Rev. Int. Contam. Ambie. 36 (1) 33-44, 2020

DOI: $10.20937 /$ RICA.2020.36.53317

\title{
MERCURIO EN UN ARROYO ALTOANDINO CON ALTO IMPACTO POR MINERÍA AURÍFERA ARTESANAL (LA RINCONADA, PUNO, PERÚ)
}

Mercury in a high altitude Andes stream with strong impact by artisanal auriffer mining (La Rinconada, Puno, Peru)

\section{Alfredo Ludwig LOZA DEL CARPIO* y Yenny CCANCAPA SALCEDO}

Facultad de Ciencias Biológicas, Universidad Nacional del Altiplano Puno, Av. Floral 1153, Puno, Perú

*Autor para correspondencia: allozade@yahoo.es

(Recibido: junio 2018; aceptado: marzo 2019)

Palabras clave: contaminación, evaluación, Lunar de Oro, cuenca del Titicaca, minería

\section{RESUMEN}

La Rinconada, en los altos Andes al sur del Perú, es una localidad dedicada fuertemente a la minería aurífera artesanal, donde relaves y desechos se vierten en arroyos de cabeceras de cuenca. Por ello se evaluó el mercurio en agua superficial y sedimentos del arroyo Lunar de Oro, en sus nacientes en el nevado Ananea. Se muestrearon cuatro puntos en los meses de diciembre de 2014, y enero y marzo de 2015, y se determinó el mercurio en ambos compartimentos por el método de espectrofotometría de fluorescencia atómica (EPA 245.2), evaluándose adicionalmente $\mathrm{pH}$, oxígeno disuelto, conductividad eléctrica, sólidos disueltos totales y temperatura mediante multiparámetro y en laboratorio. En los cuatro puntos de muestreo, el mercurio en agua presentó niveles similares, variando de $<0.00005$ a $0.00043 \mathrm{mg} / \mathrm{L}(\mathrm{n}=12)$. Según el mes, marzo presentó mayores concentraciones $(0.00034 \pm 0.000032 \mathrm{mg} / \mathrm{L})$, las cuales se relacionan con el menor caudal, superando las normas peruanas y los estándares canadienses de calidad ambiental (CEQG, por sus siglas en inglés); el pH registró un promedio general de $3.44 \pm 0.12$ y el oxígeno disuelto $2.84 \pm 0.23 \mathrm{mg} / \mathrm{L}$, valores que confirman su baja calidad. Los sedimentos tuvieron concentraciones de mercurio entre 9 y $373 \mathrm{mg} / \mathrm{kg}$, sin diferencias significativas entre zonas y meses. El $100 \%$ de las muestras superaron los límites de la NOAA(EUA) y de los CEQG. Los índices de acumulación ratificaron que el lugar se encuentra extremadamente contaminado por mercurio, y representa un alto riesgo para la salud pública y los ecosistemas involucrados.

Key words: pollution, assessment, Lunar de Oro, Titicaca basin, mining

\begin{abstract}
La Rinconada, in the high Andes at southern Peru, is a town heavily engaged in artisanal gold mining, where tailings and waste are dumped into streams of river headwaters. For this reason, mercury was evaluated in surface water and sediments of the Lunar de Oro stream, in its origins at the Ananea glacier. Four points were sampled in the months of December 2014, and January and March 2015. In both segments, mercury was determined by atomic fluorescence spectrophotometry (EPA 245.2), and pH, dissolved oxygen, electrical conductivity, total dissolved solids and temperature were determined by multiparameter and in laboratory. In the four sampling points, mercury in water presented similar levels, with values between $<0.00005$ and $0.00043 \mathrm{mg} / \mathrm{L}(\mathrm{n}=12)$,
\end{abstract}


although in March, related to a lower flow, higher levels were determined $(0.00034 \pm$ $0.000032 \mathrm{mg} / \mathrm{L}$ ), surpassing the Peruvian standards and the Canadian Environmental Quality Guidelines (CEQG); $\mathrm{pH}$ recorded an average of $3.44 \pm 0.12$ and oxygen 2.84 $\pm 0.23 \mathrm{mg} / \mathrm{L}$, values that confirmed its low quality. Sediments showed values between 9 and $373 \mathrm{mg} / \mathrm{kg}$, with no significant differences between zones and months, exceeding NOAA and CEQG limits for $100 \%$ of the samples. Pollution rates confirmed that the site is extremely contaminated by mercury, corroborating its high risk for public health and the ecosystems involved.

\section{INTRODUCCIÓN}

La minería artesanal constituye una importante actividad en países en desarrollo. Es practicada por unas 50 millones de personas en más de 70 países y está implicada en serios problemas de contaminación ambiental y salud humana (Zolnikov 2012). Sus desechos, al entrar en contacto con el agua, reaccionan y liberan metales en sus diferentes formas, además de iones como el hidrógeno que las acidifican (Quino et al. 2011). La gravedad radica en que estos metales son altamente tóxicos, biodisponibles y no biodegradables (Huayamave 2013), lo que facilita su circulación en el compartimento biótico del ecosistema (Sima et al. 2008, Ochieng et al. 2010).

El mercurio destaca entre los metales de mayor potencial tóxico (Laino-Guanes et al. 2015); es el contaminante más asociado a la minería aurífera artesanal (NIWQP 1998, Hammond et al. 2013, Díaz-Arriaga 2014) y, aunque su uso es ilegal en muchos países, todavía es extensamente utilizado en Latinoamérica, África y Asia (Veiga 1997, Hammond et al. 2013) debido a su eficiencia para la separación del oro, a su facilidad de manejo y a su bajo costo (NIWQP 1998, Donkor et al. 2015, Adjei-Kyereme et al. 2015). El mercurio no se puede descomponer ni degradar en sustancias inocuas, sólo cambia de estado y de naturaleza química, lo que implica que una vez que entra en circulación en la biosfera no desaparece (PNUMA 2005). Por ello es una de las mayores preocupaciones globales en términos de contaminación (Donkor et al. 2015).

Muchas zonas mineras son importantes fuentes de dispersión en sistemas acuáticos y contribuyen a la contaminación por metilmercurio, forma mucho más tóxica que el mercurio elemental, originada principalmente por la metilación del mercurio inorgánico a partir de bacterias (Español 2012, Segovia y Eulegelo 2015); después tiende a acumularse en organismos acuáticos (bioacumulación) en concentraciones sucesivamente mayores al ascender a niveles tróficos superiores (biomagnificación), con el riesgo de afectar a los humanos cuando consumen estos organismos (Schmitt et al. 2011, Molina et al. 2012, Hernández-Córdoba et al. 2013, LainoGuanes 2015).

En el mundo hay 10 a 15 millones de mineros artesanales que extraen el oro y producen unas $350 \mathrm{t}$ anualmente (Telmer y Veiga 2009, Veiga 2010), para lo cual utilizan unas $1000 \mathrm{t}$ de mercurio (Swain et al. 2007). Se estima que en el Perú hay de 60 a 100 mil mineros artesanales (Kuramoto 2002, Cremers y de Theije 2013), y otras 300 mil personas dependen indirectamente de esta actividad (Medina et al. 2007) que ahora se extiende por todo el país (Kuramoto 2002). Esta producción artesanal representa el $10 \%$ de la producción peruana (Medina et al. 2007, Cremers y de Theije 2013) y, considerando que en 2015 se produjeron $146 \mathrm{t}$ de oro en total (sexto lugar a nivel mundial) (USGS 2017, MINEM 2017), a la minería artesanal le corresponden 14.6 t. Si se considera que para producir $1 \mathrm{~kg}$ de oro se requieren 1.5 $\mathrm{kg}$ de mercurio (Hammond et al. 2013), la minería artesanal ha consumido unas $21.9 \mathrm{t}$ de mercurio, cuyo destino final son muchos de los recursos hídricos más importantes del Perú.

Puno es el segundo productor de oro en el país, lo cual incluye a la minería artesanal (MINEM 2017), la cual se desarrolla en las partes amazónica y altoandina. En esta última se ubica el centro minero La Rinconada (5000 msnm), que incluye los poblados La Rinconada y Lunar de Oro, donde la minería aurífera artesanal se realiza a importante escala, ya que participan en ella directamente unas 40 mil personas (Lobato 2013) de sus 82 mil habitantes (GLA 2012). Su principal impacto está asociado con el mercurio, estimándose en 15 t/año la evacuación de este metal (Mosquera et al. 1999) hacia relaves y su posterior disposición en ríos y pequeños cursos de agua que drenan en dirección a la laguna La Rinconada (Lobato 2013) y luego al lago Titicaca por intermedio del río Ramis (Gammons et al. 2006).

El riachuelo denominado Lunar de Oro nace en cabeceras de cuenca del lago Titicaca y recibe aguas 
de bocaminas y desagües del poblado del mismo nombre sin previo tratamiento (Cuentas 2006), con riesgos desconocidos para el ecosistema y la salud pública por esta causa, si se considera además que la población utiliza dicha fuente de agua para consumo y otros usos domésticos (Goyzueta y Trigos 2009). Reportes locales sobre la presencia de mercurio en aguas del río Ramis (receptor del riachuelo) señalan que los valores de este metal no exceden la normatividad (UNALM 1999, Mamani 2011, Lobato 2013, Cornejo y Pacheco 2014, DIGESA 2016); sin embargo, Gammons et al. (2006) demostraron que el mercurio en aguas de sus nacientes (en La Rinconada) sí superan los límites permitidos a nivel nacional e internacional, evidenciando con ello una delicada situación ambiental.

En este contexto, se busca contribuir al mejor conocimiento del impacto que ocasiona la actividad minera artesanal en la calidad del agua de esa parte del Perú, considerando además que para posteriores evaluaciones de riesgo es necesario previamente identificar el peligro al que están expuestas las poblaciones humanas y los ecosistemas involucrados (UNEP/WHO 2008). Se pretende también exhortar al cumplimiento de la normatividad vigente y a la instauración de un sistema de gestión ambiental orientado a corregir los daños y prevenir fatales acontecimientos futuros, que afectarían no sólo al entorno inmediato sino a zonas más bajas de la cuenca o hasta el mismo lago Titicaca, en cuyas orillas se asienta casi medio millón de habitantes (INEI 2017) que aprovechan recursos pesqueros, forrajeros, faunísticos, turísticos e incluso consumen el agua como potable, con beneficios económicos directos a la región y al país en general.

\section{MATERIALES Y MÉTODOS}

\section{Ámbito de estudio}

Se evaluaron sedimentos y agua superficial del arroyo Lunar de Oro, cuyo cauce se origina en el glaciar Ananea y atraviesa los poblados de Lunar de Oro y La Rinconada en el distrito de Ananea, provincia de San Antonio de Putina, al noreste de la región Puno, Perú (Fig. 1). La sección del arroyo en estudio se encuentra entre los 4700 y $4500 \mathrm{msnm}$ y fluye con un caudal promedio de $100.5 \mathrm{~L} / \mathrm{s}$, descargando en la laguna La Rinconada.

La zona recibe unos $707 \mathrm{~mm}$ de precipitaciones al año, frecuentemente sólidas, con una temperatura media de $1.3{ }^{\circ} \mathrm{C}$. Los meses de diciembre de 2014, y enero y marzo de 2015, durante los muestreos,

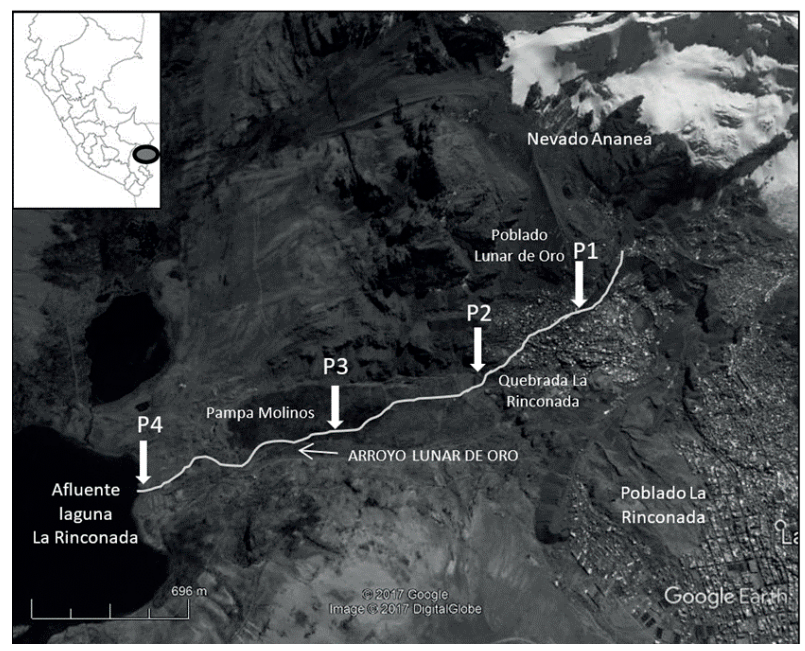

Fig. 1. Ubicación de la zona de estudio y puntos de muestreo en el arroyo evaluado (fuente: Google Earth 2017)

tuvieron precipitaciones de $105,160.8$ y $111.6 \mathrm{~mm}$, respectivamente y temperaturas máximas de $12.4^{\circ} \mathrm{C}$ y mínimas de $-3.5^{\circ} \mathrm{C}$ (SENAMHI 2017).

\section{Muestreos y análisis de muestras}

Se tomaron muestras de aguas superficiales según el Protocolo de Monitoreo de Recursos Hídricos Superficiales del Perú (ANA 2011) y sedimentos según Chen et al. (2012). Las muestras se obtuvieron en cuatro puntos del arroyo: P1, atravesando el poblado Lunar de Oro a $4732 \mathrm{msnm}$; P2, adyacente a la quebrada La Rinconada a 4720 msnm; P3, en el sector Pampa Molinos a $4655 \mathrm{msnm}$, y P4,) afluente de la laguna La Rinconada a 4557 msnm (Fig. 1). La distancia entre puntos de muestreo fue de aproximadamente $500 \mathrm{~m}$.

Para el análisis de mercurio las muestras se rotularon y colocaron en enfriadores con hielo, evitando que superaran $\operatorname{los} 2{ }^{\circ} \mathrm{C}$. Antes de $24 \mathrm{~h}$ se trasladaron al Laboratorio de Calidad Ambiental de la Universidad Mayor de San Andrés de La Paz, Bolivia. Las determinaciones se realizaron mediante el método de espectrofotometría de fluorescencia atómica (EPA, método 245.2) con un límite de determinación de $0.000050 \mathrm{mg} / \mathrm{L}$ para agua y 0.10 $\mathrm{mg} / \mathrm{kg}$ para sedimentos. Se determinaron in situ los siguientes parámetros físicos y químicos del agua: temperatura mediante termómetro digital, conductividad eléctrica (CE) y pH con multiparámetro Hach Sension 156. En laboratorio se analizaron sólidos totales disueltos (SDT) por el método gravimétrico y oxígeno disuelto por el método Winkler (APHA 1999). El caudal se estimó mediante el método del flotador (Chamorro 2011). 


\section{Análisis de datos y diseño experimental}

Se consideraron tres réplicas por cada punto de muestreo en meses diferentes, de modo que se obtuvieron un total de 12 muestras para agua superficial y 12 para sedimentos. Los datos de mercurio y los parámetros físicos y químicos y se organizaron mediante análisis de varianza (ANOVA) en diseño de bloque completo al azar, considerando los puntos de muestreo como tratamientos y los meses como bloques. Se aplicó la prueba de Tukey al encontrar diferencias significativas $(p<0.05)$. Se realizó la prueba de correlación de Pearson entre niveles de mercurio y los parámetros físicos y químicos para verificar su asociación. Para el procesamiento estadístico se utilizó el programa SPSS v. 15.

Con las concentraciones de mercurio en sedimentos se determinó el potencial de riesgo ecológico (PRE) de Hakanson (1980), calculado mediante la siguiente expresión matemática:

$P R E=R t * F C$

donde $R t$ representa el factor de respuesta tóxica para una sustancia dada, que para este caso es 40 (Hakanson 1980); $F C$ el factor de contaminación obtenido de $C_{\mathrm{Hg}} / C_{\mathrm{RP}}$, siendo $C_{\mathrm{Hg}}$ el contenido de mercurio promedio en sedimentos y $C_{\mathrm{RP}}$ el contenido referencial preindustrial $0.1 \mathrm{mg} / \mathrm{kg}$ (Cooke et al. 2011).

El $F C$ se interpretó de la siguiente manera: $F C<$ 1 bajo nivel de contaminación, $1 \leq F C<3$ moderado, $3 \leq F C<6$ considerable, $F C \geq 6$ muy alto. Los valores PERI acorde a: PERI $<40$ bajo, $40 \leq$ PERI $<80$ moderado, $80 \leq$ PERI $<160$ considerable, 160 $\leq$ PERI $<320$ alto, PERI $\geq 320$ muy alto.

Para calcular la amplitud de la contaminación por mercurio en el ámbito evaluado y la contribución antrópica al ambiente, se utilizó además el índice de geoacumulación ( $\left.I_{\mathrm{geo}}\right)$ propuesto por Muller en 1969 (Santos Bermejo et al. 2003; Islam et al. 2014), mediante la siguiente ecuación:

$I_{\text {geo }}=\log 2\left[\frac{C n}{1.5 B n}\right]$

donde $C n$ es la concentración del metal en las muestras y $B n$ el valor preindustrial 0.1. Las interpretaciones se realizaron de acuerdo con lo siguiente: < 0 prácticamente no contaminado (grado 0), 0-1 no contaminado a moderadamente contaminado (grado 1), 1-2 moderadamente contaminado (grado 2), 2-3 moderadamente a fuertemente contaminado (grado 3), 3-4 fuertemente contaminado (grado 4), fuertemente a extremadamente contaminado (grado 5) y > 5 extremadamente contaminado (grado 6).

\section{RESULTADOS Y DISCUSIÓN}

\section{Características físicas y químicas}

Los factores físicos y químicos del agua reflejan que las condiciones del arroyo Lunar de Oro tienen una muy baja calidad, atribuibles a los efectos de la actividad minera y a los asentamientos humanos de las inmediaciones, cuyas descargas ingresan directamente a éste y otros cauces (Cuadro I).

El pH alcanzó un promedio de 3.47, sin diferencias entre sectores evaluados $\left(\mathrm{F}_{3,8}=0.07 ; \mathrm{p}=\right.$ 0.974 ), valor muy por debajo de lo establecido en los Estándares de Calidad Ambiental (ECA) peruanos en la categoría 4, Conservación del Ambiente Acuático (MINAM 2017), catalogándose como aguas sumamente ácidas. Gammons et al. (2006) reportaron que las aguas de esta zona son muy ácidas (pH de hasta 3.53) debido a la oxidación de minerales sulfurosos expuestos por operaciones mineras (pirrotita, pirita y

CUADRO I. PARÁMETROS FÍSICOS Y QUÍMICOS DE AGUAS SUPERFICIALES EN CUATRO LUGARES DE MUESTREO EN EL ARROYO LUNAR DE ORO

\begin{tabular}{|c|c|c|c|c|c|c|c|c|c|c|}
\hline \multirow[b]{2}{*}{ Parámetro } & \multicolumn{7}{|c|}{ Sección del arroyo } & & \multirow[b]{2}{*}{ Media } & \multirow{2}{*}{$\begin{array}{c}\text { ECA } \\
2017 \\
\text { Cat. } 4\end{array}$} \\
\hline & $\begin{array}{c}\text { Poblado Lunar } \\
\text { de Oro }\end{array}$ & & $\begin{array}{c}\text { Quebrada La } \\
\text { Rinconada }\end{array}$ & & $\begin{array}{l}\text { Pampa } \\
\text { Molinos }\end{array}$ & & $\begin{array}{l}\text { Afluente } \\
\text { Laguna }\end{array}$ & & & \\
\hline $\mathrm{pH}$ & $3.47 \pm 0.13$ & $\mathbf{A}$ & $3.4 \pm 0.06$ & $\mathbf{A}$ & $3.47 \pm 0.18$ & $\mathbf{A}$ & $3.42 \pm 0.11$ & $\mathbf{A}$ & 3.44 & $6.5-9$ \\
\hline Temperatura $\left({ }^{\circ} \mathrm{C}\right)$ & $5.9 \pm 0.06$ & B & $6.67 \pm 0.15$ & $\mathbf{A}$ & $6.47 \pm 0.15$ & $\mathbf{A}$ & $6.23 \pm 0.12$ & $\mathbf{A}$ & 6.32 & \\
\hline $\operatorname{SDT}(\mathrm{mg} / \mathrm{L})$ & $701.67 \pm 4.41$ & A & $713.33 \pm 7.26$ & A & $701.67 \pm 7.26$ & A & $693.33 \pm 6.01$ & A & 702.5 & \\
\hline $\mathrm{OD}(\mathrm{mg} / \mathrm{L})$ & $3.28 \pm 0.06$ & A & $2.85 \pm 0.18$ & A & $2.88 \pm 0.12$ & A & $2.27 \pm 0.57$ & A & 2.82 & $\geq 5$ \\
\hline $\mathrm{CE}(\mathrm{uS} / \mathrm{cm})$ & $1391.67 \pm 4.41$ & A & $1366.67 \pm 7.26$ & A & $1403.33 \pm 18.56$ & A & $1393.33 \pm 12.02$ & A & 1388.75 & 1000 \\
\hline Caudal (L/s) & $100.67 \pm 15.87$ & A & $99.67 \pm 16.61$ & A & $101.33 \pm 16.76$ & A & $100.33 \pm 16.76$ & A & 100.55 & \\
\hline
\end{tabular}

ECA: Estándares de Calidad Ambiental peruanos; Cat.: categoría; SDT: sólidos totales disueltos; OD: oxígeno disuelto; CE: conductividad eléctrica. etras diferentes entre columnas (lugares) indican diferencias significativas a la prueba de Tuckey $(\mathrm{p}<0.05)$ para cada parámetro 
arsenopirita). Estos resultados difieren ampliamente del $\mathrm{pH}$ de las aguas de la mayoría de ríos altoandinos cuyos valores de $\mathrm{pH}$ normalmente fluctúan entre 6.93 y 8.40 (Villamarín et al. 2014).

La temperatura promedio de $6.3{ }^{\circ} \mathrm{C}$ de las aguas del arroyo es característica de un ambiente muy frío, siendo más frígidas aún en la sección más cercana al glaciar $\left(\mathrm{F}_{3,8}=7.235 ; \mathrm{p}=0.011\right)$ (Tuckey $\left.\mathrm{p}<0.05\right)$, promediando $5.9{ }^{\circ} \mathrm{C}$. Los SDT promediaron 702.5 $\mathrm{mg} / \mathrm{L}$ y no difirieron entre lugares de muestreo $\left(\mathrm{F}_{3,8}\right.$ $=1.678 ; p=0.248$ ). Estos valores se consideran elevados y evidencian la presencia de partículas y iones en abundancia, principalmente de origen inorgánico (Basha et al. 2008, Sarabia-Meléndez et al 2011). La conductividad eléctrica superó los $1300 \mathrm{uS} / \mathrm{cm}$, cuando la mayoría de los ríos altoandinos presentan promedios entre 21.45 y $143.81 \mathrm{uS} / \mathrm{cm}$ (Villamarín et al. 2014), rebasando en $40 \%$ el valor límite establecido en los ECA, lo que indica también altas concentraciones de sales, minerales y iones (Moore et al. 2008), con una elevada posibilidad de transportar metales pesados.

El oxígeno disuelto presentó valores inferiores a 3 $\mathrm{mg} / \mathrm{L}$, sin diferencias en todo el arroyo $\left(\mathrm{F}_{3,8}=1.861\right.$; $\mathrm{p}=0.214$ ), por debajo de los $5 \mathrm{mg} / \mathrm{L}$ que exige la norma y mucho más bajos aún que en la mayoría de ríos altoandinos, cuyos valores normalmente oscilan entre 8.48 y $12.58 \mathrm{mg} / \mathrm{L}$ (Villamarín et al. 2014). Normalmente los arroyos de cabeceras de cuenca tienen alto contenido de oxígeno que sólo se reduce significativamente en zonas contaminadas (Smith y Smith 2001, Molles 2006); cuando son inferioers a 3 $\mathrm{mg} / \mathrm{L}$ hay pocas probabilidades de mantener organismos fluviales (Margalef 1983), lo cual fue evidente en este arroyo al no apreciarse vestigios de vida vertebrada o invertebrada durante las inspecciones en los tramos muestreados.

\section{Mercurio en aguas superficiales}

El mercurio en todas las zonas y meses alcanzó valores desde $<0.00005 \mathrm{mg} / \mathrm{L}$ hasta $0.00043 \mathrm{mg} / \mathrm{L}$, discurriendo por el arroyo en concentraciones más o menos homogéneas durante diciembre y enero $(<$ a $0.00005 \mathrm{mg} / \mathrm{L})$, y difiriendo sólo en marzo, cuando los valores fueron desde $0.00028 \mathrm{mg} / \mathrm{L}$ en Pampa Molinos hasta $0.00043 \mathrm{mg} / \mathrm{L}$ en la quebrada La Rinconada. El promedio general fue de 0.00034 $\pm 0.000032 \mathrm{mg} / \mathrm{L}$ para este mes (Cuadro II).

Estas concentraciones de mercurio superaron el límite de $0.0001 \mathrm{mg} / \mathrm{L}$ establecido por la norma peruana (ECA categoría 4) y los $0.000026 \mathrm{mg} / \mathrm{L}$ de los estándares canadienses de calidad ambiental (CEQG 2003), sólo en los muestreos de marzo. Por ello, las
CUADRO II. CONCENTRACIONES DE MERCURIO EN AGUAS SUPERFICIALES DEL ARROYO LUNAR DE ORO SEGÚN LUGARES DE MUESTREO Y POR ÉPOCAS $(\mathrm{N}=12)$

\begin{tabular}{lccc}
\hline & Diciembre & Enero & Marzo \\
\hline Lunar de Oro & $<0.00005$ & $<0.00005$ & 0.00031 \\
Quebrada La Rinconada & $<0.00005$ & $<0.00005$ & 0.00043 \\
Pampa Molinos & $<0.00005$ & $<0.00005$ & 0.00028 \\
Afluente Laguna & $<0.00005$ & $<0.00005$ & 0.00034 \\
ECA Perú Hg (2017) & & 0.0001 & \\
CEQG Hg (2003) & & 0.000026 & \\
Caudal promedio (L/s) & 109.70 & 123.25 & 68.50 \\
\hline
\end{tabular}

Valores con fondo sombreado superan norma nacional e internacional

ECA: Estándares de Calidad Ambiental peruanos; CEQG: estándares canadienses de calidad ambiental

aguas de este arroyo constituyen un riesgo mayor para los ecosistemas acuáticos y la salud pública de la zona y de la cuenca durante este mes y en épocas secas cuando los caudales son más bajos, considerando que en marzo el promedio fue de $68.50 \mathrm{~L} / \mathrm{s}$, inferior a los meses lluviosos (Cuadro II). Gammons et al. (2006) encontraron valores de mercurio relativamente similares a los nuestros en aguas del mismo arroyo cerca del poblado Lunar de Oro, también en un mes seco, aunque sin analizar el efecto por mes ni por zonas.

Diversos estudios concluyen que en épocas secas las bajas precipitaciones e incremento de la evaporación repercuten en mayores concentraciones de mercurio en el agua, y opuestamente en épocas lluviosas hay mayor dilución y por tanto menores concentraciones (Gaete et al. 2007, Khattabi et al. 2007, Flores et al. 2010, Duman y Kar 2012, Ben Salem et al. 2014, Laino-Guanes et al. 2015), información que respalda nuestros resultados, considerando que los mayores niveles de mercurio se obtuvieron en el mes menos lluvioso (marzo).

Ello también puede justificar que las concentraciones de mercurio en tributarios de la cuenca del río Ramis disminuyen conforme discurren hasta el lago Titicaca, de modo que los valores en este lago nunca superan los $0.0001 \mathrm{mg} / \mathrm{L}$ (Gammons et al. 2006, Monroy et al. 2014, Cornejo y Pacheco 2014). Esto sugiere que en los $250 \mathrm{~km}$ de recorrido desde los nacientes de la cuenca, en La Rinconada, hasta el lago Titicaca, el mercurio se diluye progresivamente y/o sufre procesos de sedimentación y las trazas de este metal aguas abajo son indetectables (Gammons et al. 2006, Mamani 2011, Monroy et al. 2014). Las evaluaciones de DIGESA (2016) del 2007 y 2010 ratifican esta situación, ya que de sus 18 puntos de 
monitoreo (que incluyen todo el trayecto del río Ramis y ríos aportantes), sólo en dos (localizados en la laguna La Rinconada) se superaron valores de $0.0001 \mathrm{mg} / \mathrm{L}$.

\section{Mercurio en sedimentos}

Todas las muestras de sedimentos presentaron elevados valores de mercurio, desde 9 hasta $373 \mathrm{mg} / \mathrm{kg}$. El promedio más alto fue de $180 \pm 100.56 \mathrm{mg} / \mathrm{kg}$ para inmediaciones a Lunar de Oro, $75.67 \pm 42.27 \mathrm{mg} / \mathrm{kg}$ en la afluencia de la laguna La Rinconada, $50 \pm$ $27.93 \mathrm{mg} / \mathrm{kg}$ en Pampa Molinos y $43 \pm 24.02 \mathrm{mg} / \mathrm{kg}$ para la quebrada La Rinconada (Fig. 2), aunque sin diferencias significativas entre ellos $\left(\mathrm{F}_{3,6}=1.23 ; \mathrm{p}=\right.$ 0.379 ), lo que explicaría una sedimentación similar en todo el lecho del arroyo evaluado. Tampoco se evidenciaron diferencias entre meses $\left(\mathrm{F}_{2,6}=0.32 ; \mathrm{p}\right.$ $=0.737$ ), promediando $118.75 \pm 85.95 \mathrm{mg} / \mathrm{kg}$ para marzo; luego, enero y diciembre alcanzaron medias de $78 \pm 18.46 \mathrm{mg} / \mathrm{kg}$ y $64.75 \pm 14.94 \mathrm{mg} / \mathrm{kg}$, respectivamente (Fig. 3). En sus dos muestreos en el arroyo, Gammons et al. (2006) reportan valores de $228 \mathrm{mg} /$ $\mathrm{kg}$ cerca al poblado Lunar de Oro y $232 \mathrm{mg} / \mathrm{kg}$ en el sector Pampa Molinos, coincidiendo cercanamente con algunos de nuestros reportes.

En el 100\% de los muestreos las concentraciones de mercurio en sedimentos superaron ampliamente el valor de referencia de $2 \mathrm{mg} / \mathrm{kg}$, a partir del cual este metal causa efectos severos en la mayoría de los organismos bentónicos (SEL por sus siglas en inglés) o los $0.486 \mathrm{mg} / \mathrm{kg}$ que constituyen el límite de exposición para seguridad ocupacional y administración de salud (PEL) de la Administración Nacional Oceánica y Atmosférica de los Estados Unidos

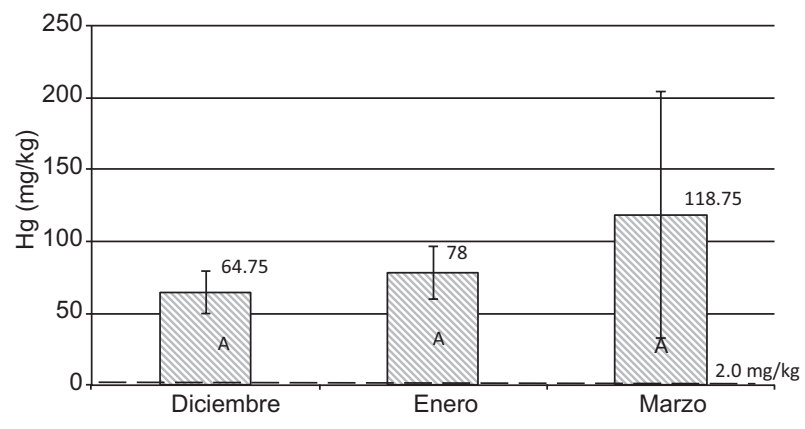

Fig. 3. Concentraciones promedio de mercurio $( \pm \mathrm{EE})$ en sedimentos del arroyo Lunar de Oro según meses, con límites establecidos por la NOAA a nivel de efectos severos (SEL). Letras iguales indican similitud estadística ( $\mathrm{p}>$ $0.05)$

(NOAA por sus siglas en inglés) (Buchman 2008). Destacan los $180 \mathrm{mg} / \mathrm{kg}$ de mercurio para el sector Lunar de Oro, que superan 186 veces lo establecido por la NOAA para SEL y 373 veces para PEL. Los CEQG (1997) establecen como guía $0.17 \mathrm{mg} / \mathrm{kg}$ de sedimentos para la protección de la vida acuática, en tanto que el programa nacional de calidad del agua para irrigación de los EUA (NIWQP 1998) indica que contenidos de $0.24 \mathrm{mg} / \mathrm{kg}$ ocasionan impactos subletales y deterioro de la capacidad reproductiva en peces. Los resultados del presente estudio superaron ampliamente estas pautas, demostrando que el arroyo evaluado mantiene en su lecho cantidades de mercurio potencialmente ecotóxicas.

El hecho de que el mercurio en agua fuera relativamente apreciable y en el sedimento mucho más notorio, sugiere que este elemento se está precipitando en el lecho y acumulándose considerablemente.

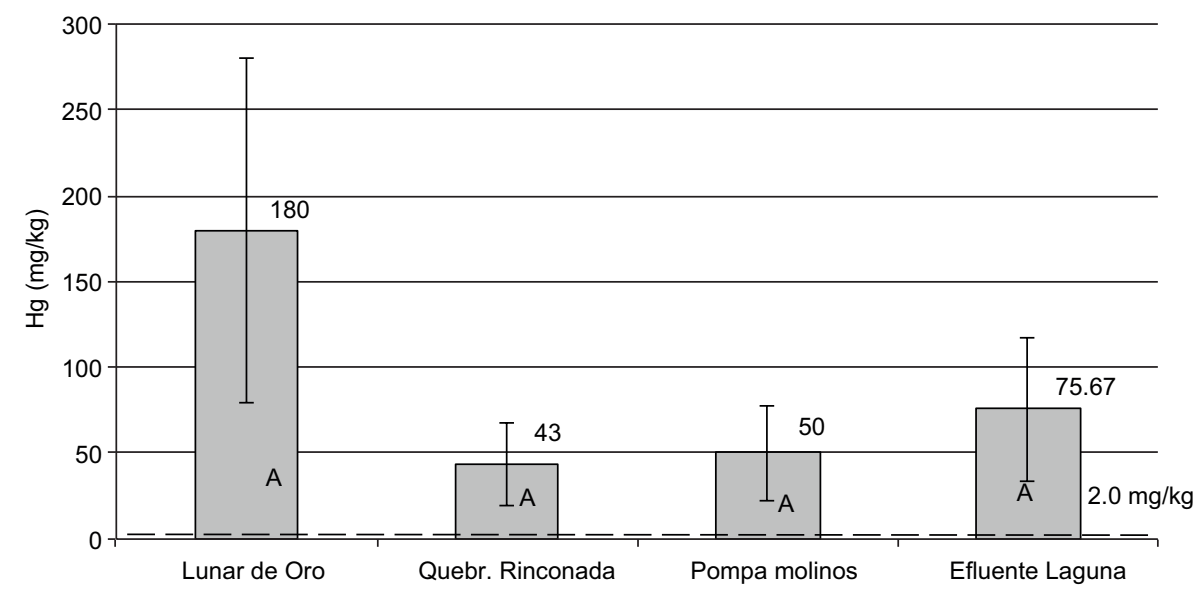

Fig. 2. Concentraciones promedio de mercurio ( \pm EE) en sedimentos del arroyo Lunar de Oro según zonas. La línea punteada indica límites de la NOAA a nivel de efectos severos $(\mathrm{SEL})$. Letras iguales sugieren similitud estadística $(\mathrm{p}>0.05)$ 
Diversos estudios corroboran la tendencia de los metales a depositarse en sedimentos con mucha facilidad y que aguas con alto riesgo de contaminación por mercurio suelen presentar concentraciones muy bajas, pero sus sedimentos evidencian niveles mucho más elevados (Szalinska et al. 2007, Leal-Ascencio et al. 2009, Laino-Guanes et al. 2015), de manera similar a lo acontecido en este estudio. Por ello Chen et al. (2012) indican que la cantidad de mercurio en sedimentos refleja mejor el grado de contaminación de un cuerpo acuático.

Es probable que el mercurio se resuspenda durante las precipitaciones y altos caudales para ser trasladado aguas abajo hasta el cauce del río Ramis, para luego depositarse en el lago Titicaca, como sugieren Mora et al. (2016). Sin embargo, Monroy et al. (2014) puntualizan que el mercurio en sedimentos de este lago es casi imperceptible $(<0.0001 \mathrm{mg} / \mathrm{kg})$, siendo relativamente importante sólo en la desembocadura del río Ramis (con $0.00078 \mathrm{mg} / \mathrm{kg}$ ); no obstante, el riesgo ecotoxicológico está latente, ya que por procesos de metilación puede circular por las redes tróficas, llegando a la ictiofauna y de allí a los seres humanos (PNUMA 2005, Cain et al. 2008, Huayamave 2013). En efecto, Monroy et al. (2014) concluyen que los peces de la zona norte del lago Titicaca (donde desemboca el río Ramis) no son recomendables para consumo humano debido a sus elevadas concentraciones de mercurio, aunque los niveles en el agua y sedimentos sean bajos.

\section{Asociación entre mercurio y parámetros físicos y químicos}

Correlaciones entre el mercurio del agua superficial y del sedimento no evidenciaron ningún nivel de asociación ( $r=0.17 ; \mathrm{p}=0.594)$ (Cuadro III); es decir, el mercurio en sedimentos no determina los niveles del agua superficial o viceversa, por tanto estos valores fueron independientes entre sí. Guzmán-Colis et al. (2011) confirman también la ausencia de correlación entre ambos valores en un estudio similar.

Las concentraciones de mercurio sí se correlacionaron significativamente con otros parámetros físicos y químicos (Cuadro III), resaltando la correlación inversa significativa entre el $\mathrm{Hg}$ del agua y el $\mathrm{pH}(\mathrm{r}=-0.752 ; \mathrm{p}=0.005)$, por lo que se infiere que bajos valores de $\mathrm{pH}$ propiciaron mayores niveles de mercurio. La mayor acidez del agua induce mayor movilidad y solubilidad del mercurio sobre todo por debajo de 3 (Spence y Barton 2003, Wu et al. 2011); además, la materia orgánica a bajo $\mathrm{pH}$ incrementa su disponibilidad para la metilación bacteriana, transformándolo en metilmercurio (Frohne et al. 2012). Es decir, aguas con bajo $\mathrm{pH}$ presentan mayor tasa de metilación y por tanto mayor bioacumulación (Jensen 1988, Shastri y Diwekar 2008); por ejemplo, lagos relativamente ácidos propician altas concentraciones de mercurio en peces (Jensen 1988), situación que eventualmente debe ocurrir en los ecosistemas implicados en esta evaluación.

Existe una importante asociación entre el mercurio del agua y los SDT $(r=0.73 ; p=0.007)$ y es probable que partículas disueltas acarreen también mercurio, por ello a mayores niveles de SDT más posibilidades de incrementar las concentraciones del metal. La correlación significativa con la conductividad eléctrica $(\mathrm{r}=0.586 ; \mathrm{p}=0.045)$ sugiere similarmente que su carga de iones y otras partículas minerales transportan también considerable carga de mercurio. Oyem et al (2014) indican que la conductividad eléctrica y los SDT del agua retienen elevada carga iónica y ambos están fuertemente correlacionados.

La alta correlación negativa entre caudal y mercurio superficial $(\mathrm{r}=-0.92 ; \mathrm{p}=0.002)$ confirmó la influencia que tiene el volumen del arroyo sobre su concentración; es decir, a menores caudales, mayores niveles de mercurio.

CUADRO III. CORRELACIONES ENTRE LAS CONCENTRACIONES DE MERCURIO DEL AGUA Y SEDIMENTOS CON LOS FACTORES FÍSICOS Y QUÍMICOS DEL AGUA SUPERFICIAL

\begin{tabular}{rcccccccc}
\hline & $\begin{array}{c}\mathrm{Hg} \\
\text { agua }\end{array}$ & $\begin{array}{c}\mathrm{Hg} \\
\text { sedimentos }\end{array}$ & $\mathrm{pH}$ & $\mathrm{T}$ & $\mathrm{SDT}$ & $\mathrm{OD}$ & $\mathrm{CE}$ & Caudal \\
\hline $\mathrm{p}$ & & 0.594 & 0.005 & 0.269 & 0.007 & 0.246 & 0.045 & 0.002 \\
\cline { 2 - 9 } Hg agua & 1 & 0.171 & $\mathbf{- 0 . 7 5 2 * *}$ & -0.347 & $\mathbf{0 . 7 3 0 * *}$ & -0.363 & $\mathbf{0 . 5 8 6 *}$ & $\mathbf{- 0 . 9 4 * *}$ \\
\hline Hg sedimentos & 0.171 & 1 & -0.217 & -0.429 & -0.004 & 0.130 & 0.016 & -0.2 \\
\cline { 2 - 9 } $\mathrm{p}$ & 0.594 & & 0.499 & 0.165 & 0.989 & 0.688 & 0.961 & 0.755 \\
\hline
\end{tabular}

$* *$ Correlación significativa $\mathrm{p}<0.01 ; * \mathrm{p}<0.05$

SDT: sólidos totales disueltos; OD: oxígeno disuelto; CE: conductividad eléctrica 
Índices de contaminación por mercurio en sedimentos

Según el índice PRE, todo el trayecto del arroyo evaluado (unos $2 \mathrm{~km}$ ) presenta "muy alto potencial de riesgo ecológico", con un promedio general de 34866.67, con valores desde 17200 hasta 72000 . El tramo en el sector del poblado Lunar de Oro alcanzó el mayor valor con un índice de 72 000, y es donde se aprecia mayor disposición de relaves y aguas residuales y mayor concentración de viviendas y establecimientos mineros. Este índice también fue importante en la desembocadura a la laguna La Rinconada (30 266.67), probablemente porque recibe todos los contaminantes evacuados desde aguas arriba (Cuadro IV).

El índice $I_{\text {geo }}$ muestra la misma situación, calificando todo el ámbito como un ecosistema "extremadamente contaminado" con mercurio, con un valor promedio de 9.18, siendo los tramos más contaminados Lunar de Oro con 10.23 y la desembocadura a la laguna con 8.97 ; los sectores adyacentes a La Rinconada y Pampa Molinos presentaron valores de 8.16 y 8.38 respectivamente (Cuadro IV).

En el cuadro $\mathbf{V}$ se comparan los índices de este estudio con los de otros ríos y arroyos relacionados a actividades de minería e industrias en diferentes partes del mundo. Resalta que aunque existen otros ecosistemas acuáticos con importantes niveles de contaminación por mercurio, ninguno supera lo acontecido en el arroyo Lunar de Oro en los valores PRE e Igeo.

\section{CONCLUSIONES}

Las características físicas y químicas de las aguas del arroyo Lunar de Oro manifestaron una muy baja calidad, propia de un ecosistema lótico completamente degradado. Su condición extremadamente ácida y los bajos niveles de oxígeno disuelto determinan que no son aptas para ningún tipo de uso en general. Los elevados niveles de SDT y conductividad eléctrica indican alta sedimentación de material particulado y presencia de sales y minerales que podrían contener diversos tipos de contaminantes. Las variaciones en las concentraciones de mercurio del agua superficial dependen de la época y magnitud del caudal del arroyo, siendo mayores en meses más secos y con menores caudales.

Las concentraciones de mercurio en sedimentos fueron extremadamente altas, superando los límites establecidos por la NOAA (SEL) y por los CEQG

CUADRO IV. POTENCIAL DE RIESGO ECOLÓGICO E ÍNDICE DE GEOACUMULACIÓN DE MERCURIO ( $\left.I_{\text {geo }}\right)$ PARA ZONAS Y TOTAL DEL ÁMBITO EVALUADO

\begin{tabular}{lccccccc}
\hline Zona & $\begin{array}{c}\mathrm{Hg} \\
(\mathrm{mg} / \mathrm{kg})\end{array}$ & $\begin{array}{c}\mathrm{FC} \\
\mathrm{Hg}\end{array}$ & $\begin{array}{c}\mathrm{PRE} \\
\mathrm{Hg}\end{array}$ & PRE & $\begin{array}{c}\text { Igeo } \\
\mathrm{Hg}\end{array}$ & $\begin{array}{c}\text { Nivel de } \\
\text { contaminación }\end{array}$ & Grado \\
\hline Lunar de Oro & 180 & 1800 & 72000 & Muy alto & 10.23 & Extrema & 6 \\
Quebrada La Rinconada & 43 & 430 & 17200 & Muy alto & 8.16 & Extrema & 6 \\
Pampa Molinos & 50 & 500 & 20000 & Muy alto & 8.38 & Extrema & 6 \\
Afluente laguna & 75.67 & 756.67 & 30266.67 & Muy alto & 8.97 & Extrema & 6 \\
Promedio & 87.17 & 871.67 & 34866.67 & Muy alto & 9.18 & Extrema & 6 \\
\hline
\end{tabular}

FC: factor de contaminación; PRE: potencial de riesgo ecológico; $\mathrm{I}_{\text {geo }}$ : índice de geoacumulación

CUADRO V. CONTAMINACIÓN POR MERCURIO EN SEDIMENTOS AFECTADOS POR MINERÍA DE ORO ARTESANAL EN OTROS RÍOS Y ARROYOS ESTUDIADOS

\begin{tabular}{lcccl}
\hline Lugar & $\begin{array}{c}\text { Sedimentos Hg } \\
(\mathrm{mg} / \mathrm{kg})\end{array}$ & Valor Igeo Hg & Valor PRE Hg & Autores \\
\hline Río Urumea, España & 62.35 & 7.9 & 9976 & Sola et al. 1990 \\
Río Kaohsiung, Taiwán & 0.35 a 1.15 & 0.3 a 3.3 & 71 a 541 & Chen et al. 2012 \\
Río Olt, Rumanía & 0.05 a 3.14 & -3.6 a 2.8 & 6.66 a 418.6 & Iordache et al. 2014 \\
Río Tano, Ghana (época seca) & 1.77 & -1 a 4 & - & Adjei-Kyereme et al. 2015 \\
Lago Chilika, India & 22.48 & 7.74 & - & Banerjee et al. 2017 \\
Arroyo Lunar de Oro, Perú & 87.17 & 9.18 & 34866.67 & Presente estudio \\
\hline
\end{tabular}

$\mathrm{I}_{\text {geo }}$ índice de geoacumulación; PRE: potencial de riesgo ecológico 
en todos los puntos de muestreo y dramáticamente en la sección Lunar de Oro. Los índices Igeo y PRE reflejan el gran riesgo ecotoxicológico para la vida silvestre y la salud pública que representa este arroyo, que constituye además una amenaza potencial para toda la cuenca en general, ya que estas cargas deben contribuir sustancialmente a las tasas de metilación y a fenómenos de bioacumulación y biomagnificación, sobre todo en receptores finales.

\section{AGRADECIMIENTOS}

Agradecemos a los ingenieros Agustín Cutipa Quenta y Jaime Chincheros por el permanente apoyo logístico brindado durante la ejecución del trabajo de campo.

\section{REFERENCIAS}

Adjei-Kyereme Y., Donkor A.K., Golow A., Yeboah P.O. y Pwamang J. (2015). Mercury Concentrations in water and sediments in rivers impacted by artisanal gold mining in the Asutifi district, Ghana. Res J. Chem. Environ. Sci. 3 (1), 40-48.

ANA (2011). Resolución Jefatural No 182-2011-ANA. Protocolo Nacional de Monitoreo de la Calidad en Cuerpos Naturales de Agua Superficial. Autoridad Nacional del Agua [en línea]. https://www.ana.gob. pe/normatividad/rj-no-182-2011-ana-0 13/10/2014

APHA (1999). Standard methods for the examination of water and wastewater (L.S. Clesceri, A. Greenberg, A.D. Eaton y M.A. Franson, Eds.). American Public Health Association/American Water Works Association/Water Environment Federation. 20th ed. Washington, EUA, 1240 pp.

Banerjee S., Pramanik A., Sengupta S., Chattopadhyay D. y Bhattacharyya M. (2017). Distribution and source identification of heavy metal concentration in Chilika Lake, Odisha India: An assessment over salinity gradient. Current Science 112 (1), 87-94.

DOI: $10.18520 / \mathrm{cs} / \mathrm{v} 112 / \mathrm{i} 01 / 87-94$

Bashaa C.A., Ghoshb P.K. y Gajalakshmi G. (2008). Total dissolved solids removal by electrochemical ion exchange (EIX) process. Electrochim. Acta 54, 474-483. DOI: 10.1016/j.electacta.2008.07.040

Ben Salem Z., Capelli N., Laffray X., Elise G., Ayadi H. y Aleya L. (2014). Seasonal variation of heavy metals in water, sediment and roachtissues in a landfill draining system pond (Etueffont, France). Ecol. Eng. 69, 25-37.

DOI: 10.1016/j.ecoleng.2014.03.072
Buchman M.F. (2008). NOAA screening quick refere Tables, NOAA OR\&R Report 08-1. Office of Response and Restoration Division, National Oceanic and Atmospheric Administration, Seattle, WA, EUA, 34 pp.

Cain A., Vannela R. y Woo K. (2008). Cyanobacteria as a biosorbent for mercuric ion. Bioresource Technol. 99 (14), 6578-6586.

DOI: 10.1016/j.biortech.2007.11.034

Chamorro G.I. (2011). Guía de hidrometría: Estimación del caudal por el método de flotadores. SENAMHI/DRLIMAN No 01-2011. Servicio Nacional de Meteorología e Hidrología, Lima, Perú, 18 pp.

Chen C.W., Chen C.F. y Dong C.D. (2012). Distribution and accumulation of mercury in sediments of Kaohsiung river mouth, Taiwan. APCBEE Proc. 1, 153-158. DOI: 10.1016/j.apcbee.2012.03.025

CEQG (1997). Canadian Environmental Quality Guidelines. Sediment Quality Guidelines for the Protection of Aquatic Life. Canadian Council of Ministers of the Environment [en línea]. http://st-ts.ccme.ca/en/index. html 25/01/2017

CEQG (2003). Canadian Environmental Quality Guidelines. Water Quality Guidelines for the Protection of Aquatic Life. Canadian Council of Ministers of the Environment [en línea]. http://ceqg-rcqe.ccme.ca/en/ index.html\#void 25/01/2017

Cooke C.A., Balcom P., Kerfoot Ch., Abbott M. y Wolfe A. (2011). Pre-Colombian mercury pollution associated with the smelting of argentiferous ores in the Bolivian Andes. Ambio 40, 18-25.

DOI: $10.1007 / \mathrm{s} 13280-010-0086-4$

Cornejo D.A. y Pacheco M. (2014). Contaminación de aguas y sedimentos por $\mathrm{As}, \mathrm{Pb}$ y $\mathrm{Hg}$ de la cuenca del río Ramis, Puno-Perú. Revista Investig. 5(4), 33-46.

Cremers L. y de Theije M. (2013). Small-scale gold mining in the Amazon. En: Small-scale gold mining in the Amazon (Cremers L., Kolen J. y de Theije M., Eds.). Board, Cuadernos del CEDLA, Amsterdam, Holanda, pp. 1-16.

Cuentas M. (2006). Evaluación cualitativa del impacto ambiental generado por la actividad minera en la Rinconada Puno. Tesis de Maestría en Gestión y Auditorías Ambientales. Facultad de Ingeniería, Universidad de Piura, Perú, 143 pp.

Díaz-Arriaga F.A. (2014). Mercurio en la minería del oro: impacto en las fuentes hídricas destinadas para consumo humano. Rev. Salud Pública 16 (6), 947957. DOI: 10.15446/rsap.v16n6.45406

DIGESA(2016). Monitoreo del río Ramis y sus tributarios, años: 2007, 2008 y 2010. Dirección General de Salud Ambiental, Ministerio de Salud [en línea]. http:// digesa.sld.pe/DEPA/vigilancia_recursos_hidricos.asp $12 / 12 / 2016$. 
Donkor A.K., Nartey V.K., Bonzongo J. y Adotey D. (2015). Artisanal mining of gold with mercury in Ghana. Res. J. Chem. Environ. Sci. 3 (1), 40-48.

DOI: $10.4314 /$ wajae.v9i1.45666

Duman F. y Kar M. (2012). Temporal variation of metals in water, sediment and tissues of the European Chup (Squalius cephalus L.). B. Environ. Contam. Tox. 89, 428-433. DOI: 10.1007/s00128-012-0679-7

EPA (1974). Method 245.2: Mercury (automated cold vapor technique) by atomic absorption. United States Environmental Protection Agency [en línea]. https:// www.epa.gov/sites/production/files/2015-08/documents/method_245-2_1974.pdf

Español S. 2012. Editorial: Contaminación con mercurio por la actividad minera. Biomédica 32(3), editorial. DOI: 10.7705 /issn.0120-4157

Flores M.L., Ramos O. y Gisberth E. (2010). Evaluación de la contaminación antrópica de las aguas del rio Jillusaya. Revista Boliviana de Química 27 (2), 75-80.

Frohne T., Rinklebe J., Langer U., Du Laing G., Mothes S. y Wennrich R. (2012). Biogeochemical factors affecting mercury methylation rate in two contaminated floodplain soils. Biogeosciences 9, 493-507. DOI: $10.5194 /$ bg-9-493-2012

Gaete H., Aránguiz F. y Cienfuegos G. (2007). Metales pesados y toxicidad de aguas del río Aconcagua en Chile. Quím. Nova 30 (4), 885-891.

Gammons C.H., Slotton D.G., Gerbrandt B., Weight W., Young C., McNearny R., Camac E., Calderon R. y Tapia H. (2006). Mercury concentrations of fish, river water, and sediment in the Río Ramis-Lake Titicaca watershed, Peru. Sci. Total Environ. 368, 637-648. DOI: 10.1016/j.scitotenv.2005.09.076

GLA (2012). Plan de Desarrollo Concertado Local al 2021. Documento Técnico. Gobierno Local de Ananea, Puno, Perú, 83 pp.

Goyzueta G. y Trigos C. (2009). Riesgos de salud pública en el centro poblado minero artesanal La Rinconada (5200 msnm) en Puno, Perú. Rev. Peru. Med. Exp. Salud Pública 26 (1), 41-44.

Guzmán-Colis G., Ramírez-López E., Thalasso F., Rodríguez-Narciso S., Guerrero-Barrera A. y AvalarGonzález F. (2011). Evaluación de contaminantes en agua y sedimentos del río San Pedro en el estado de Aguascalientes. Uciencia 27(1), 17-32.

Hakanson L. (1980). An ecological risk index for aquatic pollution control. Sedimentological approach. Water Res. 14, 975-1001.

DOI: 10.1016/0043-1354(80)90143-8

Hammond D.S., Rosales J. y Ouboter P. (2013). Managing the Freshwater Impacts of Surface Mining in Latin America. Technical Note: IDB-TN-519. InterAmerican Development Bank (IDB), 30 pp.
Hernández-Córdoba O.D., Castro-Herrera F. y Páez-Melo M. (2013). Bioacumulación de mercurio en larvas de anuros en la zona afectada por la minería de oro en el río Dagua, Buenaventura, valle del Cauca, Colombia. Acta Biol. Colomb. 18 (2), 341-348.

Huayamave J.P. (2013). Estudio de las aguas y sedimentos del río Daule, en la Provincia del Guayas, desde el punto de vista físico, químico, orgánico, bacteriológico y toxicológico. Tesis Doctoral. Programa de Doctorado en Ingeniería Ambiental y Desalinización. Universidad de las Palmas de Gran Canaria, España, 322 pp.

INEI (2017). Población 2000 al 2015. Instituto Nacional de Estadística e Informática [en línea]. http://proyectos. inei.gob.pe/web/poblacion/ 21/06/2017

Iordache M., Meghea A., Neamtu S., Popescu L. y Iordache I. (2014). Evaluation of contamination with priority hazardous substances in Olt river water and sediments near the industrial platform of Ramnicu Valcea. Rev. Chim.-Bucharest 65 (1), 87-93.

Islam M.S., Han S., Ahmed M.K. y Masunaga S. (2014). Assessment of trace metal contamination in water and sediment of some rivers in Bangladesh. Journal of Water and Environment Technology 12 (2), 109-121. DOI: $10.2965 /$ jwet.2014.109

Jensen A.L. (1988). Modelling the effect of acidity on mercury uptake by walleye in acidic and circumneutral lakes. Environ. Pollut. 50, 285-294.

DOI: 10.1016/0269-7491(88)90193-5

Khattabi H., Belle E., Servais P. y Aleya L. (2007). Variations spatiale et temporelle des abondances bactériennes dans quatre bassins de traitement du lixiviat de la décharge d'Étueffont (Belfort, France). C. R. Biologies 330, 429-438.

DOI: $10.1016 /$ j.crvi.2007.03.002

Kuramoto J. (2002). La minería artesanal e informal en el Perú. Mining, Minerals and Sustainable Development 82. IIED/WBCSD, Inglaterra, 53 pp.

Laino-Guanes R.M., Bello-Mendoza R., GonzálezEspinosa M., Ramírez-Marcial N., Jiménez-Otárola F. y Musálem-Castillejos K. (2015). Concentración de metales en agua y sedimentos de la cuenca alta del río Grijalva, frontera México-Guatemala. Tecnol. Cienc. Agua 6 (4), 61-74.

Leal-Ascencio M.T., Miranda S., Sánchez E., PrietoGarcía F. y Gordillo A. (2009). Metals pollution in the El Limon lagoon, Chiapas, Mexico. Tropical and Subtropical Agroecosystems 10 (3), 415-421.

Lobato A.L. (2013). Evaluación ambiental y programas de remediación de la cuenca alta del río Ramis. Tesis de Maestría en Ciencias con Mención en Minería y Medio Ambiente. Facultad de Ingeniería Geológica, Minera y Metalúrgica, Universidad Nacional de Ingeniería, Lima, Perú, 209 pp. 
Mamani E.N. (2011). Acumulación de mercurio en pejerrey (Basilichthys bonariensis) en hábitat norte del lago Titicaca. Tesis de Maestría en Ciencias Mención Gestión Ambiental. Facultad de Ingeniería Ambiental, Universidad Nacional de Ingeniería, Lima, Perú, 63 pp.

Margalef R. (1983). Limnología. Ediciones Omega, Barcelona, España, 1010 pp.

Medina G., Arévalo J.A. y Quea F.J. (2007). Minería ilegal en el Perú: repercusiones para el sector minero y el país. XXVIII Convención Minera, Instituto de Ingenieros de Minas del Perú, Lima, Perú, Setiembre, 107 pp.

MINAM (2017). Decreto Supremo N 004-2017-MINAM. Aprueban estándares de calidad ambiental (ECA) para agua y establecen disposiciones complementarias. Ministerio del Ambiente. Diario Oficial El Peruano, 7 de junio.

MINEM (2017). Estadística de la producción de los principales productos metálicos a nivel nacional. Ministerio de Energía y Minas, Perú [en línea]. http://www. minem.gob.pe/_estadistica.php?idSector=1\&idEstad istica $=11142$

Molina C.I., Ibáñez C. y Gibon F.M. (2012). Proceso de biomagnificación de metales pesados en un lago hiperhalino (Poopó, Oruro, Bolivia): posible riesgo en la salud de consumidores. Ecología en Bolivia 47 (2), 99-118.

Molles M. (2006). Ecología, conceptos y aplicaciones. McGraw-Hill/Interamericana de España, Madrid, España, 671 pp.

Monroy M., Maceda-Veiga A. y de Sostoa A. (2014). Metal concentration in water, sediment and four fish species from Lake Titicaca reveals a large-scale environmental concern. Sci. Total Environ. 487, 233-244. DOI: http:// dx.doi.org/10.1016/j.scitotenv.2014.03.134

Moore D., Richards G. y Story A. (2008). Electrical conductivity as an indicator of water chemistry and hydrologic process. Watershed Management Bulletin 11 (2), 25-29.

Mora A., Jumbo-Flores D., González-Merizalde M. y Bermeo-Flores S.A. (2016). Niveles de metales pesados en sedimentos de la cuenca del río Puyango, Ecuador. Rev. Int. Contam. Ambie. 32 (4), 385-397. DOI: 10.20937/rica.2016.32.04.02

Mosquera C., Trillo A. y Luján A. (1999). Propuesta para un plan de acción para el proyecto GAMA (Gestión Ambiental para la Minería Artesanal). Informe Técnico Final. Proyecto GAMA y Agencia Suiza para el Desarrollo y Cooperación COSUDE, 60 pp.

NIWQP (1998). Guidelines for interpretation of the biological effects of selected constituents in biota, water and sediment. Mercury. Information report $\mathrm{N}^{\mathrm{o}}$ 3. National Irrigation Water Quality Program, United States Department of the Interior, $113 \mathrm{pp}$.
Ochieng G.M., Seanego E.S. y Nkwonta O. (2010). Impacts of mining on water resources in South Africa: A review. Sci. Res. Essays. 5 (22), 3351-3357.

Oyem H.H., Oyem I.M. y Ezeweali D. (2014). Temperature, $\mathrm{pH}$, electrical conductivity, total dissolved solids and chemical oxygen demand of groundwater in BojiBoji Agbor/Owa área and inmediate suburbs. Research Journal of Environmental Sciences 8 (8), 444-450. DOI: $10.3923 /$ rjes.2014.444.450

PNUMA (2005). Evaluación mundial sobre el mercurio. Programa de las Naciones Unidas para el Medio Ambiente, Productos Químicos, Ginebra, Suiza, 288 pp.

Quino I., Quintanilla J. y Dalence J.S. (2011). Modelo espacio temporal hidroquímico de metales pesados dentro de la cuenca Antequera en base a la actividad minera y agrícola. Revista Boliviana de Química 28 (2), 52-62.

Santos-Bermejo J., Beltrán R. y Gómez Ariza J.L. (2003). Spatial variations of heavy metals contamination in sediments from Odiel river (southwest Spain). Environ. Int. 29, 69-77. DOI: 10.1016/S0160-4120(02)00147-2

Sarabia-Meléndez I.F., Cisneros-Almazán R., Acevesde Alba J., Durán-García M. y Castro-Larragoitia J. (2011). Calidad del agua de riego en suelos agrícolas y cultivos del valle de San Luis Potosí, México. Rev. Int. Contam. Ambie. 27 (2), 103-113.

Schmitt C.J., Stricker C.A. y Brumbaugh W. (2011). Mercury bioaccumulation and biomagnification in Ozark stream ecosystems. Ecotox. Environ. Safe. 74, 22152224. DOI: 10.1016/j.ecoenv.2011.08.008

Segovia I.F. y Eulegelo L.K. (2015). Determinar el uso de mercurio en las actividades de minería ilícita y su incidencia en componentes ambientales de agua, sedimentos e ictiofauna en la cuenca media del río Caquetá. Tesis Escuela de Postgrados de Policía Miguel Antonio Lleras Pizarro, Especialización en Gestión Ambiental. Bogotá, Colombia, 103 pp.

SENAMHI (2017). Información climática estación meteorológica Ananea. Informe de datos meteorológicos solicitados. Servicio Nacional de Meteorología e Hidrología, Puno, Perú, 2 pp.

Shastri Y. y Diwekar U. (2008). Optimal control of lake $\mathrm{pH}$ for mercury bioaccumulation control. Ecol. Model. 216, 1-17.

DOI: 10.1016/j.ecolmodel.2008.03.019

Sima M., Zobrist J., Senila M., Levei E.A., Abraham B., Dold B. y Balteneau D. (2008). Environmental pollution by mining activities. A case study in the Criş Alb Valley, Western Carpathians, Romania. Proceedings of the Swiss-Romanian Research Programme on Environmental Science \& Technology, pp. 9-20.

Smith R.L. y Smith T.M. (2001). Ecología. Pearson Educación, Madrid, España, 664 pp. 
Sola M.T., Canton L. y Millán E. (1990). Contaminación por metales pesados en sedimentos superficiales de los ríos de Guipúzcoa. Lurralde 13, 165-172.

Spence R. y Barton J. (2003). Stabilization of mercury in high $\mathrm{pH}$ tank sludges. Memorias. WM'03 Conference, Tucson, EUA, February 23-27, pp. 1 -12.

Swain E.B., Jakus P.M., Rice G., Lupi F., Maxson P., Pacyna J., Penn A., Spiegel S. y Veiga M. (2007). Socioeconomic consequences of mercury use and pollution. Ambio 36 (1), 45-61.

DOI: $10.1579 / 0044-7447(2007) 36[45:$ SCOMUA]2 .0.CO;2

Szalinska E., Haffner G. y Drouillard K. (2007). Metals in the sediments of the Huron-Erie corridor in North America: Factors regulating metal distribution and mobilization. Lakes \& Reservoirs: Research and Management 12, 217-236.

DOI: $10.1111 / \mathrm{j} .1440-1770.2007 .00339 . x$

Telmer K. y Veiga M. (2009). World emissions of mercury from artisanal and small scale gold mining. En: Mercury fate and transport in the global atmosphere (Pirrone N. y Mason R., Eds.). Springer Science, New York, EUA, pp. 131-172.

DOI: 10.1007/978-0-387-93958-2_6

UNEP/WHO (2008). Guidance for identifying populations at risk from mercury exposure. United Nations Environment Programme, Chemicals Branch/World Health Organization, Department of Food Safety, Zoonoses and Foodbourne Diseases, Ginebra, Suiza, 176 pp.

UNALM (1999). Investigación y monitoreo de los ríos Carabaya-Ramis y Cabanillas y del lago Titicaca.
Informe Técnico. Universidad Nacional Agraria La Molina, Facultad de Ciencias Forestales/Ministerio de Energía y Minas, LIMA, Perú, 62 pp.

USGS (2017). Mineral commodity summaries. Gold. U.S. Geological Survey [en línea]. https://minerals.usgs. gov/minerals/pubs/commodity/gold/mcs-2017-gold. pdf 07/06/2017

Veiga M.M. (1997). Mercury in artisanal gold mining in Latin America: facts, fantasies, and solutions. UNIDOExpert Group Meeting-introducing new technologies for abatement of global mercury pollution deriving from artisanal gold mining. Vienna, July 1-3, 23 pp.

Veiga M.M. (2010). Antioquia, Colombia: The world's most polluted place by mercury: Impressions from two field trips. University of British Columbia, Canada. Prepared for UNIDO (United Nations Industrial Development Organization), $23 \mathrm{pp}$.

Villamarín C., Prat N. y Rieradeval M. (2014). Caracterización física, química e hidromorfológica de los ríos altoandinos tropicales de Ecuador y Perú. Lat. Am. J. Aquat. Res. 42 (5), 1072-1086. DOI: 10.3856/vol42issue 5-fulltext-12

Wu H., Ding Z., Liu Y., Liu J., Yan H., Pan J., Li L., Lin H., Lin G. y Lu H. (2011). Methylmercury and sulfatereducing bacteria in mangrove sediments from Jiulong River Estuary, China. J. Environ. Sci. 23, 14-21. DOI: $10.1016 / \mathrm{S} 1001-0742(10) 60368-3$

Zolnikov T.R. (2012). Limitations in small artisanal gold mining addressed by educational components paired with alternative mining methods. Sci. Total Environ. 419, 1-6. DOI: 10.1016/j.scitotenv.2012.01.017 